\title{
Pharmacological Interventions against Obesity: Current Status and Future Directions
}

\author{
Bernd Schultes \\ eSwiss Medical \& Surgical Center, St. Gallen, Switzerland
}

\author{
Keywords \\ Metabolic syndrome - Appetite - Weight loss · \\ GLP-1 analogs
}

\section{Summary}

The metabolic syndrome (MS) comprises a cluster of metabolic disturbances including obesity, hyperglycemia, dyslipidemia, and elevated blood pressure that are pathophysiologically highly interlinked and that altogether lead to an increased cardiovascular (CV) risk. While lifestyle interventions represent the basic therapeutic measure to address all components of the MS, they are in most cases not effective enough to reduce the CV risk. Therefore, pharmacological interventions are necessary for many patients. This review will provide an overview on current and potential future pharmacological interventions for obesity, which is the main driver of the MS.

(c) 2016 S. Karger GmbH, Freiburg

\section{Introduction}

The term 'metabolic syndrome' (MS) was initially introduced as a pathophysiological concept in which insulin resistance is forming the central part and which describes the frequently observed concurrent prevalence of different cardiometabolic risk factors like obesity, hyperglycemia, dyslipidemia, and elevated blood pressure. During the last years, obesity was increasingly recognized as the main driver of insulin resistance and, thus, the components of the MS. Therefore, weight loss represents a key goal in the treatment of overweight and obese patients with MS. However, long-term weight loss is difficult to achieve and in most cases not great enough to efficiently reduce the risk of cardiovascular (CV) events. This has been impressively demonstrated by the large Look AHEAD trial in which more than 5,000 overweight or obese pa- tients with type 2 diabetes mellitus (T2DM) were randomized to an intensive lifestyle intervention that promoted weight loss and increased physical activity or to receive regular diabetes support and education [1]. Despite a greater weight loss in the intensive lifestyle intervention group than in the other group ( 6.0 vs. $3.5 \%$; $<<0.001$ ) after a mean follow-up of 9.6 years as well as a better glycemic control and initial improvement in fitness, no differences in CV event rates were found between the two groups. On the one hand, this result is rather disappointing; on the other hand, it also stresses the need of pharmacological interventions to effectively reduce the CV risk. This review will provide an overview on current and possible future pharmacological interventions for obesity, which is the main driver of many other components of the MS.

\section{Lessons Learned from Pharmacological Anti-Obesity Studies}

The history of anti-obesity, weight loss-promoting drugs has been a history of many hopes and disappointments [2]. Many of the respective drugs had to be withdrawn from the market a few years after their approval for obesity treatment because of severe adverse side effects that were mostly identified in post-marketing studies. Among these were fenfluramine and dexfenfluramine, both of which are amphetamines that increase the central nervous release of serotonin. The drugs were withdrawn from the market in the late 1990s because it turned out that they provoked vascular heart disease or pulmonary hypertension in some patients. About 10 years later, sibutramine, a serotonin and noradrenaline reuptake inhibitor, as well as rimonabant, an endocannabinoid receptor blocker, were withdrawn from the market since sibutramine was found to increase the risk of CV events and rimonabant the risk of depression and suicide. After these negative experiences general concerns have been raised referring to all anti-obesity drugs. Fueled by these concerns, several new anti-obesity drugs have not been approved by the regulatory authorities due to a lack of longterm data on potential side effects and the overall risk-benefit ratio.

\section{KARGER}

() 2016 S. Karger GmbH, Freiburg

Fax +497614520714 
Obesity is now increasingly regarded as a complex disease with not only a strong genetic background but also important psychosocial influences. Evidence has been accumulated showing that body weight is highly regulated, involving many redundant neuroendocrine pathways. Most of the current anti-obesity drugs act on distinct appetite regulatory centers in the brain. However, the effectiveness of single agents in regard to body weight reduction is often limited, probably due to the great redundancy of the regulatory system. Also, it has been found that, to date, all anti-obesity drugs lose their weight-reducing effect once the medication is stopped. Interestingly, most placebo-controlled pharmacological anti-obesity studies have drawn a rather stereotypical picture of body weight course during the intervention no matter which particular drug was tested (fig. 1). Here, the respective anti-obesity drug exerts weight-reducing effects that exceed the weight loss achieved under the placebo condition due to the regularly undertaken background lifestyle intervention. The maximal amount of weight loss is usually achieved about 6-9 months after the start of the intervention. Thereafter, the weight-reducing effect of the respective drug is more or less preserved but usually no further body weight reduction is achieved. If the drug is stopped, the body weight rapidly increases and reaches the level of the placebo group within months. Based on this stereotypical pattern of findings, it is now clear that to date all anti-obesity drugs only have a limited effect on body weight which is in most cases fully established after 6-9 months and that the respective drug needs to be taken continuously to preserve this limited effect. In consequence, pharmacological obesity treatment usually means long-term treatment which is not surprising when considering obesity as a chronic disease.

\section{Orlistat}

The only anti-obesity drug that has been on the market for almost 20 years now is orlistat. It is also the only drug that does not affect appetite. Orlistat is a lipase inhibitor that reduces the digestion and thus absorption of dietary fat. The most common side effect is steatorrhea, in particular after fatty meals, which often leads patients to stop taking the medication. As has been shown in a large randomized, placebo-controlled trial, the average effect of the drug is a weight loss of about $4 \mathrm{~kg}$ after 1 year of treatment [3]. This weight loss is achieved on top of the weight loss due to a background dietary intervention (orlistat vs. placebo: $10.2 \%$ (10.3 kg) vs. $6.1 \%(6.1 \mathrm{~kg}) ; \mathrm{p}<0.001)$ that in this study comprised a reduction of minimal caloric intake of $500 \mathrm{kcal}$ per day.

\section{Liraglutide}

Liraglutide is a drug that has recently been approved for obesity treatment in Europe. It is an analog of glucagon-like peptide 1 (GLP-1), i.e. a peptide hormone that is released from neuroendocrine L-cells of the intestinal tract. The substance has already been well known as an anti-diabetes drug for several years. GLP-1 exerts

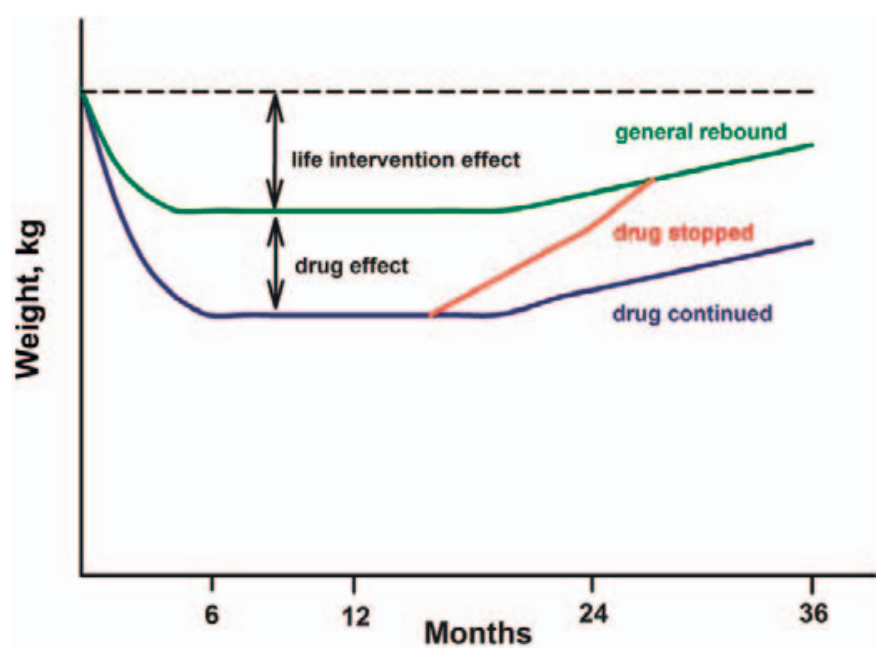

Fig. 1. Schematic body weight course as observed in many pharmacological studies on anti-obesity drugs.

the so-called incretin effect on beta-cell insulin secretion after oral food intake, thereby reducing postprandial blood glucose levels. The weight-reducing effects of liraglutide are currently believed to mainly derive from an appetite-reducing effect of GLP-1 receptor agonism that takes place at the level of the vagal nerve which then transmits the anorectic signal via the nucleus tractus solitarius to the brain. While GLP-1 receptors have recently also been identified in the human brain $[4,5]$, it is still not clear whether GLP-1 analogs administered to the subcutaneous fat enter and thus directly affect the brain. However, a recent neuroimaging study that applied the functional magnetic resonance imaging (MRI) technology showed that liraglutide treatment reduces the neuronal activity response to food pictures in the insula and putamen and enhances the satiating effect of meal intake and concurrent neuronal response in the putamen and amygdala [6]. A similar study showed likewise that liraglutide treatment decreases the activation of the parietal cortex in response to highly desirable food pictures [5].

Clinical studies on the effect of liraglutide on body weight in non-diabetic, obese patients have revealed a clear-cut dose-response relationship, with the greatest weight loss achieved with the highest, i.e. $3 \mathrm{mg}$ per day, liraglutide dose [7]. A similar dose-response relationship was also found in a study that included overweight or obese subjects with T2DM [8]. Against the background of these findings, a large randomized, placebo-controlled study was carried out in 3,731 non-diabetic, overweight or obese patients. The patients were randomized in a 2:1 ratio to receive $3.0 \mathrm{mg}$ liraglutide per day or placebo as applied by a daily subcutaneous injection over a study period of 56 weeks. All included patients additionally received counseling on lifestyle modification as a background treatment. At the end of the 1-year treatment period, the patients in the liraglutide group had lost on average $8.4 \mathrm{~kg}$ whereas those of the placebo group had lost $2.8 \mathrm{~kg}(\mathrm{p}<0.001) .63 .2 \%$ of the patients in the liraglutide group and $27.1 \%$ in the placebo group had lost at least $5 \%$ of their body weight $(\mathrm{p}<0.001)$. The percentage of patients who lost at least $10 \%$ was 33.1 and $10.6 \%$, respectively $(\mathrm{p}<0.001)$. Importantly, liraglutide treatment also showed 
favorable effects on other components of the MS, i.e. a reduction in glucose, lipids, and blood pressure levels.

The most common side effects of liraglitude are mild or moderate nausea and diarrhea which in most cases vanished over time. However, it should also be mentioned that liraglutide as well as other GLP-1 receptor agonists increase the risk of cholecystolithiasis and probably also of pancreatitis. Also, liraglutide is known to slightly increase the resting heart rate which has previously raised concerns about its CV safety. Of note in this context, a recent large liraglutide trial in T2DM patients has documented a reduction of CV events under treatment with $1.8 \mathrm{mg}$ liraglutide when compared with placebo treatment [9]. Naturally, it remains unknown whether this beneficial effect can be extrapolated to the much higher dose of $3.0 \mathrm{mg}$.

While the results of the liraglutide weight loss trials appear to be very promising, it should also be noted that the financing of this kind of obesity treatment remains an unresolved problem. The highest currently approved liraglutide dose for T2DM treatment is $1.8 \mathrm{mg}$, and that dose is already quite expensive. Considering the huge T2DM market for liraglutide, it appears unlikely that the pharmaceutical company will reduce the price of the substance in order to make the $3.0 \mathrm{mg}$ liraglutide dose affordable for most obese patients. In this context, it should also be taken into account that in most countries the insurance companies do not cover the cost for pharmacological obesity treatment yet.

In general, the concept of using GLP-1 receptor agonists for obesity treatment appears to be very attractive considering that other GLP-1 analogs have already entered the market for T2DM treatment. Some of these analogs show a much longer action and therefore need to be injected only once a week [10, 11], which could help patients that have problems with more frequent injections. Furthermore, a new formulation of the GLP-1 receptor agonist semaglutide, which can be taken orally, is currently under investigation (ClinicalTrials.gov identifier: NCT02863419). It is clear, however, that all of these GLP-1 receptor agonists need to be thoroughly tested for obesity treatment in large clinical trials that include non-diabetic, obese patients before the respective drugs can be used in clinical practice.

\section{Naltrexone / Bupropion SR}

A combination of the two substances naltrexone and bupropion in a slow-release (SR) formulation has recently been approved for weight loss by the European Medicines Agency (EMA). It is now on the European market under the trade name Mysimba ${ }^{\circledR}$; in the USA its trade name is Contrave ${ }^{\circledR}$. Naltrexone is an opioid receptor antagonist while bupropion inhibits the reuptake of the neurotransmitters dopamine and noradrenaline. Bupropion alone is also used as an antidepressant and for smoking cessation. The combination of these two drugs has been shown to exert a synergistic effect on body weight reduction. The standard fixed combination includes $8 \mathrm{mg}$ naltrexone and $90 \mathrm{mg}$ bupropion, which can be taken up to four times per day. While the substances are primarily believed to exert their appetite-suppressing effect at a hypothalamic level $[12,13]$, neuroimaging studies have shown that the combination drug also affects higher brain centers [14]. Specifically, naltrexone/bupropion attenuated to hypothalamic activation during watching a food cues video but enhanced the activation of brain regions involved in inhibitory control mechanism (anterior cingulate), internal awareness (superior frontal, superior parietal, insula), and memory function (hippocampus).

In a large randomized, placebo-controlled study, 1,742 overweight or obese patients were randomized to receive $32 \mathrm{mg}$ naltrexone combined with $36 \mathrm{mg}$ bupropion, $16 \mathrm{mg}$ naltrexone combined with $360 \mathrm{mg}$ bupropion, or placebo [15]. The average weight loss was $-6.1,-5.0$, and $-1.3 \%$, respectively, after 1 year. A weight loss of at least $5 \%$ was achieved by $48 \%$ of the patients in the $32-\mathrm{mg}$ naltrexone group, by $39 \%$ in the $16-\mathrm{mg}$ naltrexone group, and by $16 \%$ in the placebo group (both $\mathrm{p}<0.001$ vs. placebo). The most frequent adverse side effect of the combined drug treatment was nausea which was reported by $29.8 \%$ of the patients in the $32-\mathrm{mg}$ naltrexone group, $27.2 \%$ in the $16-\mathrm{mg}$ naltrexone group, and $5.3 \%$ in the placebo group. Other reported adverse effects of the treatment were headache, constipation, dizziness, vomiting, and dry mouth. Furthermore, while in this study no tendency towards more depression and suicidality were identified, other studies on the combination drug suggested that it could increase these specific risks [16].

The long-term safety of the naltrexone/bupropion combination therapy should have been assessed in a large randomized, placebocontrolled trial including 8,910 overweight or obese patients. Unfortunately, the sponsor of the study has released a confidential interim analysis to the public so that the academic leaders of the study recommended termination of the trial. The data obtained until the time point of termination were recently published and provided no signal for an increased CV risk under the medication [17]. However, the early termination and, accordingly, limited statistical power of the trial clearly precludes ruling out all safety concerns.

\section{Lorcaserin}

Lorcaserin is a specific serotonin $2 \mathrm{C}$ receptor agonist that is currently not approved in Europe but in the USA for anti-obesity treatment. The trade name of the substance is Belviq ${ }^{\circledR}$, and it is taken orally with a regular dose of $10 \mathrm{mg}$ twice per day. It is assumed to act on anorexigenic neurons within the hypothalamus, thereby reducing appetite. A recent neuroimaging study that used the functional MRI technology showed a reduction of attentionrelated brain area activations in response to food cues involving the parietal and visual cortex as well as the insula and amygdala in obese patients [18].

In one large randomized, placebo-controlled trial that included 3,182 overweight and obese patients, lorcaserin treatment resulted in an average weight loss of $5.8 \mathrm{~kg}$ after 1 year as compared with a weight loss of $2.2 \mathrm{~kg}$ in the placebo group ( $\mathrm{p}<0.001$ ) which only received dietary and exercise counseling [19]. $47.5 \%$ of the patients in the lorcaserin group and $20.3 \%$ in the placebo group achieved a 
weight loss of at least $5 \%(\mathrm{p}<0.001)$; the percentage of patients achieving a weight loss of at least $10 \%$ was 22.6 and $7.7 \%$, respectively ( $\mathrm{p}<0.001)$. It is noteworthy, however, that the study was limited by a very high dropout rate of about $45 \%$ in the lorcaserin group and $55 \%$ in the placebo group. Only a minority of patients left the study because of adverse side effects, though.

Based on the previous negative experience with the other serotonin agonists fenfluramine and dexfenfluramine, echocardiographic examination was systematically carried out and did not indicate an increased incidence of vascular heart disease or pulmonary hypertension [19]. Common side effects of the drug are headache and vertigo but overall the substance appears to be well tolerated. However, it is important to note that the substance must not be taken together with other serotonergic drugs in order to avoid the development of a serotonin syndrome. Long-term safety data on the drug are still missing which is likely the reason why the drug is still not available on the European market. Of note, the EMA has already rejected an approval application for the drug once.

\section{Phentermine / Topiramate ER}

The combination of the sympathomimetic amine (amphetamine) phentermine that increases the release of dopamine and noradrenaline with the AMPA receptor antagonist topiramate in an extended-release (ER) form is approved for weight loss treatment in the USA but not yet in Europe. Its trade name is Osymia ${ }^{\circledR}$. Of note, topiramate is on the European market for anticonvulsive and migraine treatment and is often used in much higher doses than for anti-obesity treatment in combination with phentermine. The maximal doses for weight loss treatment approved by the Food and Drug Administration is $75 \mathrm{mg}$ phentermine combined with $92 \mathrm{mg}$ topiramate ER, but also a lower dose of $75 \mathrm{mg}$ and $46 \mathrm{mg}$, respectively, is available. The idea behind the combination of the two substances is to reduce potential adverse side effects while using its synergistic effects on appetite suppression.

In a large randomized, placebo-controlled trial [20], 2,487 patients were treated with two different doses of the combination drug or placebo for 1 year. Patients receiving the higher drug dose lost on average $10.2 \mathrm{~kg}$ as compared to $1.4 \mathrm{~kg}$ in the placebo group ( $p<0.001$ ). A weight loss of at least $5 \%$ was achieved by $70 \%$ of patients of the high-dose group but only by $21 \%$ of patients of the placebo group $(\mathrm{p}<0.001)$. The respective figures for at least $10 \%$ weight loss were 48 and $7 \%(\mathrm{p}<0.001)$. Side effects were quite common, including paresthesia (21\% of the patients in the highdose group vs. $2 \%$ in the placebo group), constipation (17 vs. $3 \%$ ), insomnia (10 vs. $5 \%$ ), dizziness (10 vs. $3 \%$ ), and dysgeusia (10 vs. 1\%). Furthermore, depression-related adverse events were also more common in patients receiving the drugs than in the placebo group ( 7 vs. $4 \%$ ), as were anxiety-related adverse events ( 8 vs. $3 \%$, respectively). Based on these findings and still missing long-term safety data, the EMA has already rejected approval applications for the drug combination twice so that it remains unclear whether this therapeutic will be ever available in Europe in the future.

\section{Future Directions}

While several new drugs for obesity treatment are available now, their overall effectiveness appears to be generally limited and markedly weaker than that of bariatric surgery, which represents another therapeutic option for severely obese patients. Of note, bariatric surgery has brought many new factors and mechanisms to light that play a critical role in the regulation of body weight and thus represent novel potential therapeutic targets for obesity treatment. Beside the enhancement of the GLP-1 signal which is seen after gastric bypass surgery and that can, at least in part, be mimicked pharmacologically by administration of GLP-1 receptor agonists, biliary acid metabolism and gut microbiota composition may represent other promising new targets for pharmacological interventions [21]. However, considering the redundancy of the body weight regulating system it appears likely that, in general, combination therapies will be more effective than the administration of a single agent.

Clearly, the continuously increasing knowledge on the pathophysiology of obesity and the MS will bring about new targets for pharmacological intervention. Nevertheless, in the light of previous disappointing experiences it needs to be emphasized that all new drugs need to undergo thorough clinical testing before they can be introduced to the market. In particular, long-term safety data are of utmost importance since it can always be possible that drug-related adverse side effects offset the beneficial effects of weight loss in the long run.

Individual responses to different drugs also represent an important issue that should be more closely investigated in the future. Regarding most drugs, it has been recognized that some patients show an excellent weight loss response whereas others show no response at all. Here, identifying factors that predict individual drug responses will help to better guide the pharmacological therapy, thereby getting closer to a personalized therapeutic approach.

\section{Conclusion}

During the last years, several new anti-obesity drugs have emerged and promise some help for those patients suffering from obesity. However, the effectiveness of these drugs is limited, and long-term safety remains largely unknown in many cases. Putting this forward, more research is clearly needed to more effectively address obesity as a main driver of the MS.

\section{Disclosure Statement}

The author received lecture fees and advisory honoraria from the following pharmaceutical companies: Eli Lilly, NovoNordisk, AstraZeneca, Boehringer Ingelheim, MSD, and Sanofi. 


\section{References}

1 Look AHEAD Research Group; Wing RR, Bolin P, Brancati FL, et al: Cardiovascular effects of intensive lifestyle intervention in type 2 diabetes. $\mathrm{N}$ Engl J Med 2013;369:145-154.

2 Carter R, Mouralidarane A, Ray S, Soeda J, Oben J: Recent advancements in drug treatment of obesity. Clin Med (Lond) 2012;12:456-460.

3 Sjostrom L, Rissanen A, Andersen T, Boldrin M, Golay A, Koppeschaar HP, Krempf M: Randomised placebocontrolled trial of orlistat for weight loss and prevention of weight regain in obese patients. European Multicentre Orlistat Study Group. Lancet 1998;352:167-172.

$\checkmark 4$ Ten Kulve JS, van Bloemendaal L, Balesar R, RG IJ Swaab DF, Diamant M, la Fleur SE, Alkemade A: Decreased hypothalamic glucagon-like peptide-1 receptor expression in type 2 diabetes patients. J Clin Endocrinol Metab 2016;101:2122-2129.

5 Farr OM, Sofopoulos M, Tsoukas MA, Dincer F, Thakkar B, Sahin-Efe A, Filippaios A, Bowers J, Srnka A, Gavrieli A, Ko BJ, Liakou C, Kanyuch N, Tseleni-Balafouta S, Mantzoros CS: GLP-1 receptors exist in the parietal cortex, hypothalamus and medulla of human brains and the GLP-1 analogue liraglutide alters brain activity related to highly desirable food cues in individuals with diabetes: a crossover, randomised, placebo-controlled trial. Diabetologia 2016;59:954-965.

6 Ten Kulve JS, Veltman DJ, van Bloemendaal L, Barkhof F, Drent ML, Diamant M, IJzerman RG: Liraglutide reduces CNS activation in response to visual food cues only after short-term treatment in patients with type 2 diabetes. Diabetes Care 2016;39:214-221.

7 Astrup A, Rossner S, Van Gaal L, Rissanen A, Niskanen L, Al Hakim M, Madsen J, Rasmussen MF, Lean ME: Effects of liraglutide in the treatment of obesity: a randomised, double-blind, placebo-controlled study. Lancet 2009;374:1606-1616.
Davies MJ, Bergenstal R, Bode B, Kushner RF, Lewin A, Skjoth TV, Andreasen AH, Jensen CB, DeFronzo RA: Efficacy of liraglutide for weight loss among patients with type 2 diabetes: the SCALE diabetes randomized clinical trial. JAMA 2015;314:687-699.

9 Marso SP, Daniels GH, Brown-Frandsen K, Kristensen P, Mann JF, Nauck MA, Nissen SE, Pocock S, Poulter NR, Ravn LS, Steinberg WM, Stockner M, Zinman B, Bergenstal RM, Buse JB: Liraglutide and cardiovascular outcomes in type 2 diabetes. N Engl J Med 2016;375:311-322.

10 Blonde L, Jendle J, Gross J, Woo V, Jiang H, Fahrbach JL, Milicevic Z: Once-weekly dulaglutide versus bedtime insulin glargine, both in combination with prandial insulin lispro, in patients with type 2 diabetes (AWARD-4): a randomised, open-label, phase 3, noninferiority study. Lancet 2015;385:2057-2066.

11 Marso SP, Bain SC, Consoli A, Eliaschewitz FG, Jódar E, Leiter LA, Lingvay I, Rosenstock J, Seufert J, Warren ML, Woo V, Hansen O, Holst AG, Pettersson J, Vilsbøll T; SUSTAIN-6 Investigators: Semaglutide and cardiovascular outcomes in patients with type 2 diabetes. N Engl J Med 2016;DOI: 10.1056/NEJMoa1607141.

12 Greenway FL, Dunayevich E, Tollefson G, Erickson J, Guttadauria M, Fujioka K, Cowley MA: Comparison of combined bupropion and naltrexone therapy for obesity with monotherapy and placebo. J Clin Endocrinol Metab 2009;94:4898-4906.

13 Greenway FL, Whitehouse MJ, Guttadauria M, Anderson JW, Atkinson RL, Fujioka K, Gadde KM, Gupta AK, O’Neil P, Schumacher D, Smith D, Dunayevich E, Tollefson GD, Weber E, Cowley MA: Rational design of a combination medication for the treatment of obesity. Obesity (Silver Spring) 2009;17:30-39.

14 Wang GJ, Tomasi D, Volkow ND, Wang R, Teland F, Caparelli EC, Dunayevich E: Effect of combined naltrexone and bupropion therapy on the brain's reactivity to food cues. Int J Obes 2014;38:682-688.
5 Greenway FL, Fujioka K, Plodkowski RA, Mudaliar S, Guttadauria M, Erickson J, Kim DD, Dunayevich E: Effect of naltrexone plus bupropion on weight loss in overweight and obese adults (COR-I): a multicentre, randomised, double-blind, placebo-controlled, phase 3 trial. Lancet 2010;376:595-605.

16 Nuffer W, Trujillo JM, Megyeri J: A comparison of new pharmacological agents for the treatment of obesity. Ann Pharmacother 2016;50:376-388.

17 Nissen SE, Wolski KE, Prcela L, Wadden T, Buse JB, Bakris G, Perez A, Smith SR: Effect of naltrexonebupropion on major adverse cardiovascular events in overweight and obese patients with cardiovascular risk factors: a randomized clinical trial. Jama 2016;315: 990-1004.

18 Farr OM, Upadhyay J, Gavrieli A, et al: Lorcaserin administration decreases activation of brain centers in response to food cues and these emotion- and salience-related changes correlate with weight loss effects: a four week long randomized, placebo-controlled, double-blinded clinical trial. Diabetes 2016;DOI: 10.2337db16-0635.

19 Smith SR, Weissman NJ, Anderson CM, Sanchez M, Chuang E, Stubbe S, Bays H, Shanahan WR: Multicenter, placebo-controlled trial of lorcaserin for weight management. N Engl J Med 2010;363:245-256.

20 Gadde KM, Allison DB, Ryan DH, Peterson CA, Troupin B, Schwiers ML, Day WW: Effects of lowdose, controlled-release, phentermine plus topiramate combination on weight and associated comorbidities in overweight and obese adults (CONQUER): a randomised, placebo-controlled, phase 3 trial. Lancet 2011;377:1341-1352.

21 Bray GA, Frühbeck G, Ryan DH, Wilding JP: Management of obesity. Lancet 2016;387:1947-1956. 